\title{
The Application of Distributed Raptor Codes in Cooperative Transmission System
}

\author{
Wang Chao \\ Zhengzhou Electromechanical Engineering Research Institute, China \\ 854909839@qq.com
}

\begin{abstract}
Keywords: Cooperative Communications; Relay Transmission; Digital Fountain Codes; Distributed Raptor Codes.
\end{abstract}

\begin{abstract}
In order to reduce computational complexity and transmission delay in the cooperative transmission system based on digital fountain codes, the paper proposes the cooperative communication scheme based on a distributed Raptor codes. The throughput is treated as the parametric analysis of the system complexity and the link packet loss rate is looked as parametric analysis of the transmission delay of the system, Moreover, the distributed Raptor codes compares with LT codes and other digital fountain codes transmission scheme. Theoretical analysis and simulation results indicate that the Raptor codes in throughput and decoding success rate is more greatly improved than LT codes. When the link packet loss rate is big, this distributed Raptor codes cooperative transmission scheme can greatly improve the performance of the system on the transmission delay and the larger link deletion probability is, obviously the greater advantages is.
\end{abstract}

\section{Introduction}

Cooperative communication is one of the hot issues concerned by the academic circles in the field of wireless communication in recent years, the idea is through in the mutual coordination between terminals in a wireless communication network, which combats wireless channel fading and improves the reliability of communication and the transmission range [1]. In the study of cooperative communication for the moment, it is mainly designed from the two aspects of the physical layer and network layer transmission scheme. The physical layer generally uses space diversity technology and the traditional fixed rate coding scheme (namely the repetition code, turbo code and space time codes, etc.), this can obtain diversity gain and coding gain, so as to improve the transmission performance of the system [2 5]. Using fixed rate coding transmission needs to known the sender or has been an accurate estimate of the

Channel state information (CSI) in order to make the system achieve the best trade-off point of validity and reliability. However, the state information generally varies with time and space in the real wireless channel, transmission scheme of fixed rate coding is difficult to make transmission system achieve optimal, and cooperative communication systems usually have a plurality of transmission links, by passing to sending feedback information to accurately estimate all transmission channel state information is almost impossible. From the point of view of the network layer, the two hop cooperative relay transmission scheme design should meet the following several characteristics: (1) due to the independence between the source / relay link and relay / destination node link, we must ensure the two hop links are reliable transmission; (2) we as much as possible reduce the data transmission delay between point to point, especially for delay sensitive applications. Existing solutions typically use Forward Error Correction (FEC) to transmit redundant code package, combined with hybrid automatic retransmission request hybrid automatic repeat request (HARQ) to transmit redundant code package, this ensure the reliability of communication, at the same time we can greatly reduce the number of retransmissions. Scholars have designed various kinds of FEC codes, such as RS codes and Tornado codes. In order to be able to adapt to changing channel conditions and making full use of the channel capacity, John Byers and Michael Luby in 1998 proposed concept of digital fountain [6], it is proposed a kind of ideal solution for the large-scale data distribution and reliable broadcasting application 
characteristics. So it is very suitable for the application of fountain codes to the cooperative communication system which has a plurality of transmission links.

In the literature [7], to use the independent digital fountain codes ensures the transmission reliability of each hop in two hops. But in these schemes, every relay requires decoding and re-encoding the received data, and it should transmit an Acknowledgement (ACK) to the source to confirm the correct reception at each time. Obviously, this will bring the relay node large computational complexity, and due to frequent feedback information, it can cause large propagation delay. Cascade code was used in the literature [8 9], namely relay nodes received fountain code package from the source before the second code without the need for decoding. But all the cooperative transmission based on concatenated code will cause the destination node decoding complexity. In Ref. [10] proposed the concept of decomposition LT codes (DLT), which is in a certain extent reduces the overhead of decoding and encoding and decoding complexity, but in bad transmission environment, the error rate is high. Liau [11] presents a kind of soliton - like rateless coding (SLRC) scheme for two source single relay Y type network, as a whole, making the degree distribution be close to the solitary waves in the distribution, so that BP decoding algorithm can decode efficiently, and its performance is better than that of DLT code, but to adapt to the channel capacity is poor and decoding complexity degree is higher.

In order to further ensure the reliability of communication at the same time, reduce the complexity of encoding and decoding and bit error rate, this paper proposes a distributed cooperative transmission scheme based on Raptor codes, distributed cooperative communication system are given for the three ends of the two layer encoding degree distribution function design, analyzes the transmission delay of encoding and decoding of Raptor codes the throughput, and the success rate of the system, and based on the direct transmission scheme based on Raptor codes and Raptor decoding relay forwarding scheme comparison, theoretical analysis and simulation results show that, the distribution of cooperative transmission mechanism of fountain codes, can significantly improve the system performance of the transmission delay, and packet loss rate the greater the more obvious advantage. The scheme is suitable for single relay cooperative network and multi - stage relay system.

\section{Distributed Raptor Code}

Although the LT code encoding and decoding is simple, the decoding error probability is the existence of a high pan effect, and the complexity of coding and packet length is nonlinear, it is not suitable for long code. Therefore, Shokrollahi [12] puts forward another improved fountain code, called Raptor code. Raptor code is a concatenated code, which generally uses Low density parity check (LDPC) code that is high rate and Hamming code as the outer code or pre-coding, with an average degree of small weak LT codes as inner codes, decoding the coding process as shown in Figure 2. The corresponding Raptor codes can also be divided into two steps, first using BP decoding to recover intermediate symbols, then using the traditional decoding method of correcting decoding. To intermediate symbols because pre-coding has some error correction capability, relaxing requirements for the LT code, so the overall decoding complexity is reduced to linear complexity, and overcomes the effect of error floor of LT codes, are superior in performance of error rate and cost.

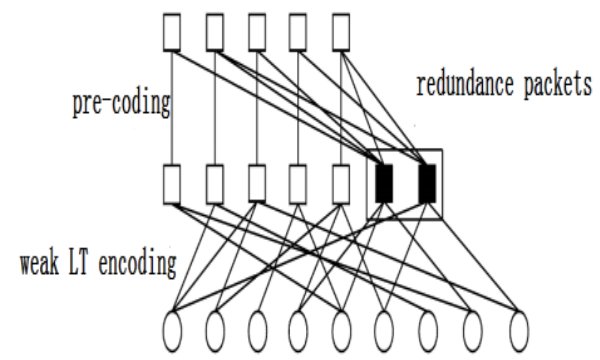

Fig.1 Raptor code encoding process

In order to correct the defects of distributed LT code [13]. This paper presents a coding scheme of distributed Raptor code. Raptor code first uses a grouping code as pre-coding, and then uses the weakening LT codes which the average is about 3 to encode the data symbols. The decoding method is 
simple, and the overhead of decoding and encoding complexity are relatively small. Transmitter is random linear coding for the original k packets, and the receiver only needs to receive to $\mathrm{N}=\mathrm{k}(1+\varepsilon)$ groups ( $\mathrm{N}$ was slightly larger than $\mathrm{k}$ ), can with great probability correctly restore the original packet, $\varepsilon$ here said the receiver correctly decoding need receive redundancy. For the Raptor code, the key to obtain high decoding success probability is to design a good degree distribution function. In the literature [14] Luby designs a good performance of the distribution function of the degree of RSD (robust soliton distribution) $\mu(x)=\sum_{i=1}^{k} \mu_{i} x^{i}$, of which the probability is $\mathrm{d}=\mathrm{i}, \mathrm{K}$ is the number of source file input symbols.

Unlike DLT, DRaptor code treats LDPC code as pre-coding, then in the stage of LT code through a two layer random code to generate a code of each packet, as shown in Figure 2:

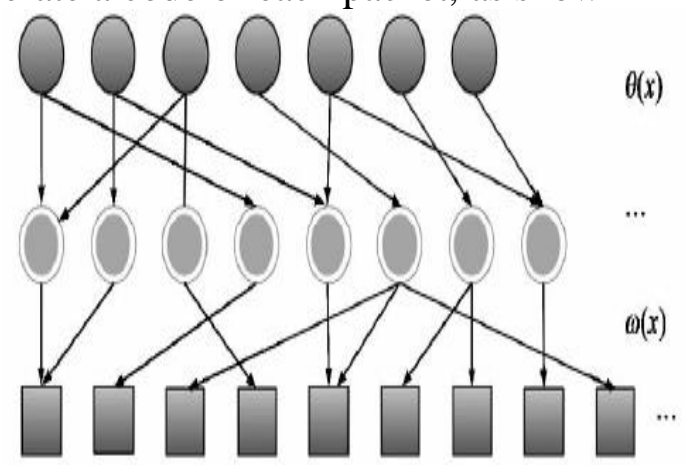

Fig.2 DRaptor coding block diagram with two layer encoding degree distribution function

Suppose you want to transmit initial K input symbol, DRaptor coding process is described as follows:

(1) In the first layer, K input symbols use LT coding according to the random degree distribution function $\theta(x)$, code output packet denotes as DR-1 data packet;

(2) The DR-1 data packet is treated as the second layer random encoder input, and LT code according to the degree distribution function $\omega(x)$. The final output of the encoding package calls DR-2 data packets.

DRaptor decoding uses single BP (Belief Propagation) algorithm for Raptor decoding, and greatly reduces the delay and complexity. In order to achieve BP good decoding performance, DRaptor code is the key to design two appropriate distribution function [15] $\theta(x)$ and $\omega(x)$.

\section{System Model}

The typical three terminal cooperative communication system as a model, as shown in Figure 3. Source nodes in the assistance of $\mathrm{R}$ relay nodes transfer the information to the destination node $\mathrm{D}$. Assumed the channel between nodes is erasure channel, corresponding to the deletion probability: $P_{\mathrm{e}}^{S D}, P_{e}^{S R}, P_{e}^{R D}$. $\mathrm{S}$ and $\mathrm{R}$ are coding of Raptor to data packets which is being sent. The source $\mathrm{S}$ selects degree distribution function $\theta(x)$, and encodes data to send before $\mathrm{R}$ and $\mathrm{D}$ radio. The data packets where $\mathrm{R}$ receives from $\mathrm{S}$ does not need the decoding, instead to choose another degree distribution function of the received data encoding is performed again and the encoded data packet sent to the D. D according to the received data packet uses BP algorithm for decoding, and decodes after transmitting messages to the $\mathrm{S}$ and $\mathrm{R}$ when $\mathrm{S}$ and $\mathrm{R}$ stop sending. In order to get a good decoding performance, the key is to design two suitable degree distribution functions $\theta(x)$ and $\omega(x)$ which makes degree distribution function $\mu(x)$ of the $\mathrm{D}$ received data packets has better decoding performance, for example, is approximately equal to the RSD. 


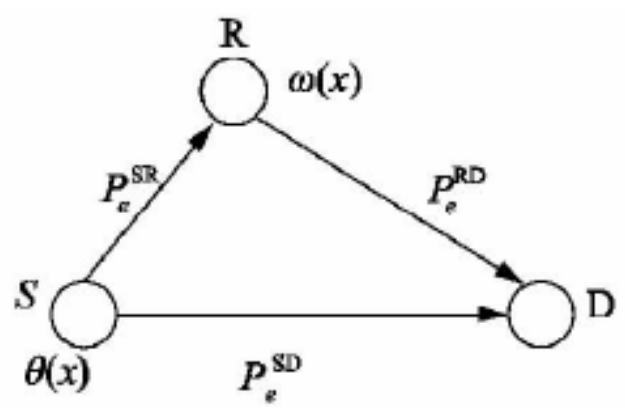

Fig. 3 The three end of the cooperative communication system model

\section{Parameters Analysis Of Raptor Codes}

\subsection{Block size}

Raptor codes in erasure channel coding and decoding complexity is $\mathrm{O}(\mathrm{K} \log (1 / \varepsilon))$, where $\varepsilon$ is the overhead of decoding, $\mathrm{K}$ input symbols into data blocks, complexity with code length increased, and LT codes encoding complexity is $\mathrm{O}(\log \mathrm{K})$, decoding complexity is $\mathrm{O}(\mathrm{K} \log \mathrm{K})$ and complexity as the code length logarithmic increase, so the Raptor codes in coding and decoding complexity is decreased.

In the comparative analysis, select the block size $\mathrm{k}=32,64,128,256,512,1024$ byte, block size decides the entire source data throughput. If the selected block is very large, then the recipient is required for a long time to wait for receiving enough the coded symbols to start decoding. And the choice of a small piece, although a single block coding decoding costs less time , and receiver doesn't need big delay for decoding, but overhead which they can take in data transmission will become great.

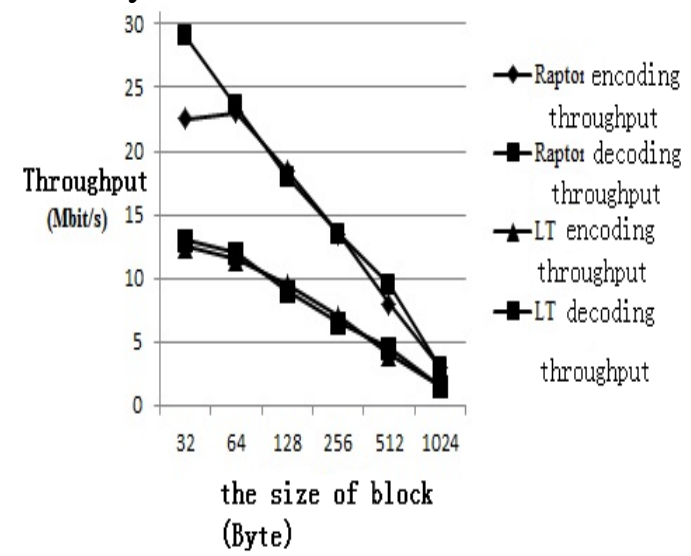

Figure 4. Encoding and decoding throughput

Figure 4 shows a different block size effect on system throughput, which the x-axis for block size, respectively, the value of $\mathrm{k}=32,64,128,256,512,1024$ bytes. From figure we can see that with increasing block size, encoding and decoding throughput shows a decreasing trend, and roughly a linear decline. With increasing block size, throughput is getting smaller and smaller, because in the coding and decoding process of fountain codes, relatively large block matrix for inverse complexity is very high, so in order to improve the coding / decoding speed, small block is more favorable. But the choice of block is not too small ( $\mathrm{k}$ is less than or equal to 32), if the block's selection is too small, it is caused by context switching overhead will greatly increase, thus affecting the coding and decoding performance. Raptor codes in coding and decoding speed is LT coding and decoding speed of document [5] compared with obvious advantages, basically can be increased by two times.

\subsection{The number of symbol erasure}

Fountain codes are a non systematic code, when the encoder output of the coded symbols in the transmission process appeared lost, damaged, resulting in receiver decoding failure, the receiver needs to receive more symbols, and this time received symbols called erasure symbol. Ideally, you only need to receive $\mathrm{K}$ symbols can be decoded, but that in the actual situation of the network is not up to, due to network packet loss and need to accept $\mathrm{K}(1+\varepsilon)$ symbols to decode correctly, $\varepsilon$ is overhead ratio, the smaller $\varepsilon$ is, the higher code / decoder performance is, the greater the effective throughput of the 
network is. According to the different probability of erasure channel, received correct number of symbols erasure is not the same. In order to test the relationship between $\mathrm{K}$ and $\mathrm{E}$, the assumed an erasure channel probability $\mathrm{p}$ is 0.05 , and raptor code generates the symbol to $5 \%$ probability of random deletion to simulated by deleting the probability $\mathrm{p}=0.05$ erasure channel, the $\mathrm{K}=10,100,1000$ and 2000 , $\mathrm{L}=1024$ bytes of experiment, the experimental results as shown in figure 5 .

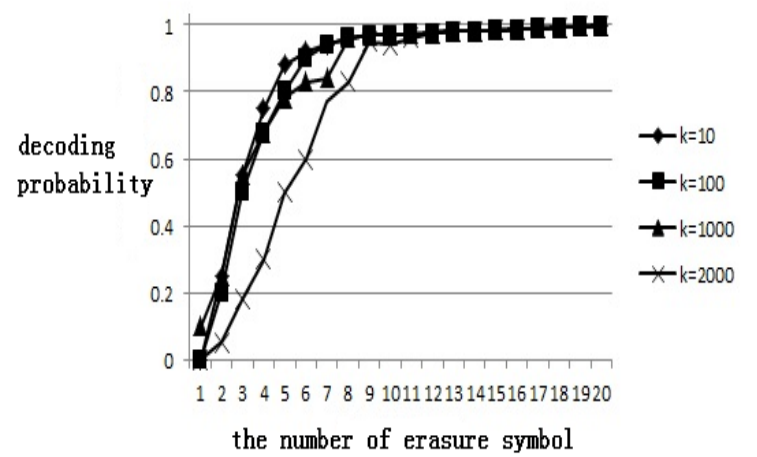

Figure 5. Raptor codes required for decoding erasure symbol

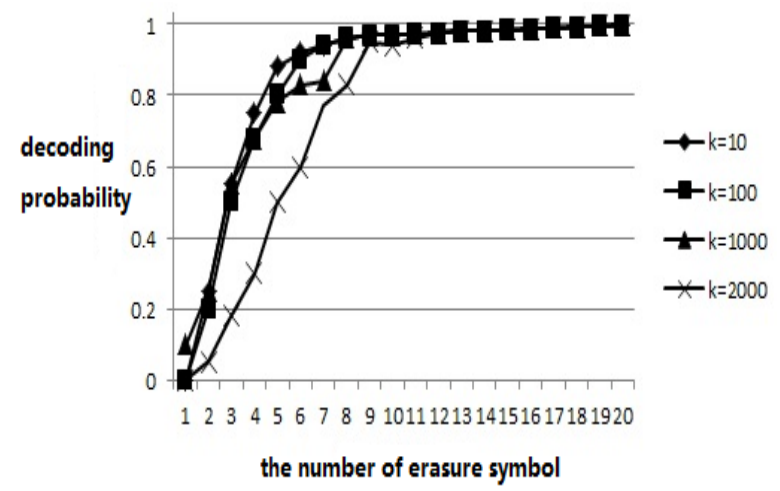

Figure 6. LT codes required for decoding erasure symbol

Figure 5 can be seen, the coded symbol K between the 10 2000 receives end in multiple receive nine erasure symbol, successful decoding probability reached $99 \%$, i.e. overhead rate $\varepsilon<=1 \%$, different $\mathrm{K}$ values obtained curves coincide, indicating that the decoding of Raptor codes are in good agreement, even if the $\mathrm{K}$ is very large, just nine erasure symbol can reach $99 \%$ of the decoding success rate. In the design of the upper application, $\mathrm{N}=\mathrm{K}+9$ can be set to a general threshold. Figure 6 is LT fountain codes decoding success rate and overhead as a result, reaching $99 \%$ of the decoding success rate threshold needs $\mathrm{N}=\mathrm{K}+13$, so Raptor code overhead rate is obviously less than that of the LT code overhead rate, in the same packet loss probability ,Raptor codes can significantly improve the effective bandwidth utilization.

\section{PERFORMANCE SIMULATION}

Presented in this section uses distributed Raptor codes for the three protocol transmission delay of cooperative transmission protocol DR-CC, raptor codes direct transmission protocol RA-DT and Raptor codes based relay forwarding cooperative transmission protocol (RA-CC) . Monte Carlo simulation of each source node transmits 500 packets, and the results are compared with the theoretical analysis. Assuming that the ACK feedback signal can guarantee error free transmission, and does not consider the relay compiled code, such as processing time. Target distribution function of the degree of choice RSD degree distribution $\mu(x)$, parameters were $\mathrm{k}=500, \mathrm{c}=0.03, \delta=0.05$. The degree distribution decomposition algorithm to decompose $\mu(x)$, which $D_{\theta}$ is $50, D_{\omega}$ is 10 . 

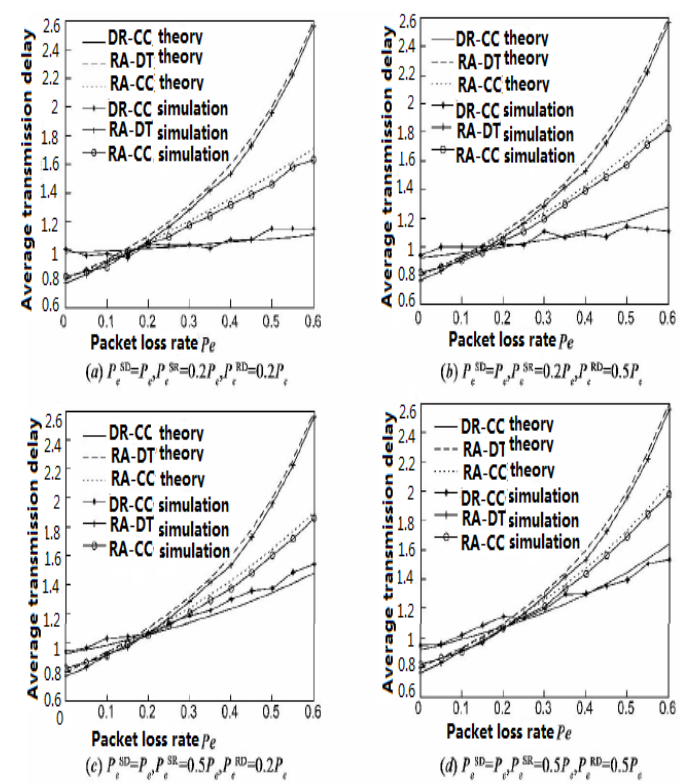

Figure 7 .The change curve of the average transmission delay with packet loss rate of the channel system

Figure 7 (a), (b), (c), (d) the numerical simulation results and the average transmission delay of 3 kinds of different protocol link conditions are given. Decoding overhead $\varepsilon$ is 0.2 . As can be seen in various channel conditions direct transmission protocol and decode and forward cooperation protocol simulation results and numerical results are in good agreement. For distributed Raptor codes for cooperative transmission protocol, may have a certain deviation due to the value of the transmission coefficient $\mathrm{A}$, the simulation results and the numerical results basically are the same, but there are some deviations.

From the figure can also be seen when each link of the packet loss rate is very small (approximately less than 0.2), protocol with direct transmission and relay decoding turn that The performance of the protocol is almost the same, the transmission delay is less than the distributed cooperative transmission protocol based on Raptor code. Mainly because of the packet loss rate is very small, in based on distributed Raptor codes for cooperative transmission protocol in the destination node respectively from the direct link and relay cooperative link received data packet of high repetition rate, so the gain is smaller. When the link packet loss rate is large, based on distributed Raptor codes for cooperative transmission protocol has the best performance, decode and forward cooperative protocol is second, direct transport protocol performance is the worst. Moreover, with the increase of the link loss rate, the advantage of distributed cooperative transmission protocol based on Raptor code is more obvious. In addition, effect of source relay link on system performance is larger, when the source relay link packet loss rate is relatively smaller, the better the system performance is.

\section{Conclusion}

In wireless networks, when the sending the channel is unknown circumstances, the fountain codes can adaptively achieve matching rate and time varying channel capacity so that we can achieve efficient and reliable communication. The characteristics of fountain codes and excellent performance make it very suitable for cooperative communication in wireless relay networks. To two relay cooperative transmission link in the digital fountain code so as to keep the communication reliability while reducing the computational complexity and delay. In this paper, the distributed Raptor codes used in the cooperative communication system, are given for in three terminal cooperative communication systems with distributed two layer encoding and code degree distribution function design, raptor codes and decoding throughput, success rate and system transmission delay is analyzed, and based on Raptor codes direct transmission schemes and based on Raptor codes for decode and forward cooperative scheme are compared. The results of the analysis show that the Raptor code is lower than LT codes in computing degree, decoding success rate is higher, and when the link packet loss rate is larger when using cooperative transmission scheme in distributed Raptor codes requires smallest transmission delay. 
Moreover, with the increase of packet loss rate, the advantage of cooperation scheme based on the distributed Raptor code is more obvious. The three point type single antenna system as a model, but the proposed scheme combined with relay selection strategy also applies to multiple single antenna relay forwards two hop system or combined with space-time coding for three point type multi antenna system model.

\section{References}

[1] Jung M C,Kwak J S,Kim H S,et al. Adaptive subcarrier, bit, and power allocation algorithm for MIMO-OFDMA system [C].In Proc. of IEE VTC 2014-Spring,Milan ,Italy ,May,2014.

[2] Zhao P, Kuang L, Lu J. Carrier frequency offset estimation using extended Kalman filter in uplink OFDMA systems[C].InProc.IEEEICC'2006,Istanbul,Turkey,June,2006.

[3] Sezginer S,Bianchi P. Asymptotically efficient reduced-complexity frequency offset estimation for uplink MIM0-OFDMA systems[C].In Proc. IEEE ICC'07,Glasgow,Scotland, August, 2007.

[4] Kim K J, Pun M, Reid T, et al. Joint frequency offset and channel estimation for UL- MIMOOFDMA systems using the parallel Schmidt Kalman filters[C].In Proc.IEEE ICASSP'2007,Haw ai' I,USA,May,2007.

[5] Mahler R P S. Multitarget Bayes filtering via first-order multi-target Moments[J].IEEE Trans. on Aerospace and Electronic Systems,2013,39 (4):1152-1178.

[6] Doucet A,Freitas N,Murphy K,et al. Rao-blackwellised particle filtering for dynamic Bayesian networks[C].The 16th Annual Conference on Uncertainty in Artificial Intelligence,San Francisco, CA, 2010.

[7] Lei Wei Jia ,Xie Xian-zhong,Li Guang-jun. The scheme and performance of wireless cooperative relay system using digital fountain codes[J].Acta Electronica Sinica,2010,38 (1) :228-233. (in Chinese)

[8] R Gummadi, R S Sreenivas. Relaying a fountain code across multiple nodes [A]. Proceedings of Information Theory Workshop (ITW) [C].Porto,Portugal:IEEE Press,2008.149-153.

[9] A Tarable,I Chatzigeorgiou,I J Wassell. Randomly select and forward:Erasure probability analysis of a probabilistic relay channel model [A] . Proceedings of Information Theory Workshop (ITW) [C] . Taormina, Italy:IEEE Press,2009.41- 45.

[10] Rui Cao, Liuqing Yang. Decomposed LT codes for cooperative relay communications [J]. IEEE Journal on selected areas in communications,2012,30(2):407-414.

[11] Liau A, Yousefi S,Il-Min K. Binary soliton-like rateless coding for the Y-Network[J].IEEE Transacions on Communications, 2011,59(12):3217-3222.

[12] Shokrollahi A. Raptor codes[J]. IEEE Transactions on Information Theory, 2006, 52(6): 25512567.

[13] GUO Feng. Wireless sensor network data distributed storage based on Raptor code. Applied Science and Technology,2014,10,41(5):040-04.

[14] M Luby. LT codes [A]. Proceeding of IEEE Foundations of Computer Science (FOGS) [C]. Vancouver, Canada:IEEE Press, 2002.271-280.

[15] GAO Fei, ZENG Xian-feng, BU-Xiang-yuan. Improved Scheme for Short-Length Raptor Codes Based on Frequency Hopping Communication [J]. Transactions of Bei jing Institute of Technology, 2013,4,33(4):03-05. 